\title{
Hidrocoloides como estabilizantes en bebidas de maíz dulce (Zea mays var. saccharata) y gel de aloe vera (Aloe barbadensis Miller)
}

\section{Hydrocolloids as stabilizers in beverages from sweet corn (Zea mays var. Saccharata) and aloe vera gel (Aloe barbadensis Miller)}

Karen Paola Contreras-Lozano'; Héctor José Ciro-Velásquez²; Julio Cesar Arango-Tobón ${ }^{3}$

\begin{abstract}
${ }^{1}$ Ing. Agroindustrial, MSc., Estudiante de Doctorado. Universidade Federal de Lavras. Lavras - Minas Gerais, Brasil; e-mail: kpcontreras|@gmail.com; (D) https://orcid.org/0000-0002-5237-1490

${ }^{2}$ Ing. de Alimentos, Ph.D. Universidad Nacional de Colombia, sede Medellín, Facultad de Ciencias Agrarias, Departamento Ingeniería Agrícola y Alimentos. Medellín - Antioquia, Colombia; e-mail: hjciro@unal.edu.co; Dhttps://orcid.org/0000-0002-4398-0470
\end{abstract}

${ }^{3}$ Ing. Agrícola, Ph.D. Universidad Nacional de Colombia, sede Medellín, Facultad de Ciencias Agrarias, Departamento Ingeniería Agrícola y Alimentos. Medellín - Antioquia, Colombia; e-mail: jcarango@unal.edu.co; Dhttps://orcid.org/0000-0003-1625-0541

Cómo citar: Contreras-Lozano, K.P.; Ciro-Velásquez, H.J.; Arango-Tobón, J.C. 2019. Hidrocoloides como estabilizantes en bebidas de maíz dulce (Zea mays var. saccharata) y gel de aloe vera (Aloe barbadensis Miller). Rev. U.D.C.A Act. \& Div. Cient. 22(2):e1090. http:// doi.org/10.31910/rudca.v22.n2.2019.1090

Artículo de acceso abierto publicado por Revista U.D.C.A Actualidad \& Divulgación Científica, bajo una licencia Creative Commons CC BY-NC 4.0

Recibido: Noviembre 22 de 2018

Aceptado: Agosto 28 de 2019

Editado por: Rita María Ávila Gómez de Hernández

\section{RESUMEN}

La sedimentación de partículas es un fenómeno común en dispersiones alimentarias, que está asociado con una calidad deficiente y se puede controlar usando diferentes aditivos, dentro de los cuales, se destacan el uso de hidrocoloides. Se evaluó el efecto de la concentración de goma xantano $(0,025-0,075 \%)$, carboximetilcelulosa sódica $(0,025-0,075 \%)$ y gel de aloe vera $(0,5-$ $1,5 \%$ ), en las propiedades fisicoquímicas, sensoriales y estabilidad a la sedimentación, en bebidas elaboradas a partir de maíz dulce. No se observó variación significativa en el contenido de sólidos solubles totales, $\mathrm{pH}$, densidad, acidez titulable, potencial $\zeta$, tamaño de partículas, velocidad de sedimentación y parámetros de color con las concentraciones empleadas de hidrocoloides y de aloe vera. El potencial $\zeta$ indicó estabilidad ligera en las bebidas $(>-30 \mathrm{mV})$ y la floculación de la emulsión, lo que se evidencia en la distribución del tamaño de partícula $(1-600 \mu \mathrm{m})$. La viscosidad y la estabilidad a la sedimentación aumentaron con concentraciones $>0,05 \%$ de GX y CMC, reflejándose en una mayor valoración sensorial de la apariencia. El aumento en la concentración de aloe vera no mostró efecto en la estabilidad de las bebidas.

Palabras clave: carboximetilcelulosa; goma xantano; estabilidad; suspensiones; viscosidad (CAB Thesaurus). 


\section{ABSTRACT}

Particle sedimentation is a common phenomenon in food dispersions, which is associated with poor quality and can be controlled using different additives within which the use of hydrocolloids stands out. The effect of the concentration of xanthan gum $(0.025-$ $0.075 \%$, sodium carboxymethylcellulose $(0.025-0.075 \%)$ and aloe vera gel $(0.5-1.5 \%)$, on physicochemical, sensory properties and sedimentation in beverages made from sweet corn, were evaluated. No significant variation was observed in the content of total soluble solids, $\mathrm{pH}$, density, titratable acidity, $\zeta$ potential, particle size, sedimentation index and color parameters with the concentrations of hydrocolloids and aloe vera. The potential $\zeta$ indicated slight stability in beverages $(>-30 \mathrm{mV})$ and emulsion flocculation, which is evidenced in the particle size distribution $(1-600 \mu \mathrm{m})$. The viscosity and sedimentation stability increased with concentrations $>0.05 \%$ of GX and CMC, reflected in a higher sensory assessment of appearance. The increase in aloe vera concentration showed no effect on the stability of the beverages.

Keywords: carboxymethylcelulose; xanthan gum; stabilizers; suspensions; viscosity (CAB Thesaurus).

\section{INTRODUCCIÓN}

El maíz dulce (Zea mays L. var. saccharata) es una variedad con características sensoriales agradables de sabor, de textura y de aroma, lo cual, es un aliciente para su uso en la obtención de alimentos procesados. Este producto presenta una escasa transformación industrial, consumiéndose, principalmente, como hortaliza fresca, en alimentos precocidos o en forma de grano entero en conserva (Szymanek et al. 2015).

La estabilidad de dispersiones y las suspensiones alimenticias pueden estar influenciadas por cambios en el tiempo, debido al comportamiento de los componentes inmiscibles que pueden presentar separación de fases y frente a las interacciones indeseables entre los componentes individuales, es decir, las interacciones físicas entre las partículas dispersas (Drapala et al. 2018).

Los hidrocoloides son polímeros hidrofílicos, con una amplia gama de propiedades funcionales favorables para su aplicación en dispersiones alimentarias, ya que pueden cambiar la reología y la microestructura del medio, para obtener productos estables, con propiedades sensoriales aceptables (Norton et al. 2011). La aplicación de hidrocoloides, como GX y CMC, ha sido reportada previamente en bebidas elaboradas con frutas y hortalizas, para evitar la sedimentación y controlar la vida útil (Abbasi \& Mohammadi, 2013; Abedi et al. 2012; Aghajanzadeh et al. 2017; Akkarachaneeyakorn \& Tinrat, 2015; Nwaokoro \& Akanbi, 2015). Una bebida formulada con la pulpa extraída de los granos de maíz dulce contiene una gran variedad de componentes, como partículas sedimentables y fases inmiscibles, acuosa y oleosa, que pueden generar un comportamiento inestable, dando lugar a un detrimento de su calidad, durante el almacenamiento.
El aloe vera (Aloe barbadensis Miller) es una planta que contiene en sus hojas un gel mucilaginoso, que ha generado gran interés en la industria alimentaria, por la presencia de más de 200 constituyentes diferentes, de los cuales, 75 tienen actividad biológica, como componentes nutritivos, fenólicos y fitoesteroles, propiedades que convierten al gel de aloe vera (AV) en un ingrediente potencial en la formulación de alimentos, aplicado en bebidas (Elbandy et al. 2014; Sánchez-Machado et al. 2017).

Debido a que la aplicación del maíz dulce en alimentos procesados, específicamente en bebidas, ha sido poco explorada y los estudios son incipientes, el objetivo del presente trabajo fue desarrollar bebidas a partir de maíz dulce, evaluando el efecto de la concentración de GX, CMC y AV en la estabilidad a la separación de fases, las propiedades fisicoquímicas y la evaluación sensorial.

\section{MATERIALES Y MÉTODOS}

Formulación y elaboración de las bebidas. Para la elaboración de las bebidas, se utilizó maíz dulce (Z. mays var. saccharata) y leche condensada comercial, para el aporte de sólidos solubles; gel de $\mathrm{AV}$, como ingrediente nutricional; dos tipos de hidrocoloides, como estabilizantes, goma xantano (GX) y carboximetilcelulosa sódica (CMC) y, como conservantes, benzoato de sodio y sorbato de potasio, en las concentraciones permitidas y establecidas en la Norma Técnica Colombiana - NTC 3549/1999 y la Res. 7992/1991. Para la formulación de las bebidas, 20\% de pulpa obtenida de la molienda de los granos y $12 \%$ de leche condensada fueron dispersados en agua, hasta obtener una concentración de sólidos solubles totales de $10^{\circ}$ Brix. La mezcla fue sometida a un tratamiento térmico a $72^{\circ} \mathrm{C}$, por 30 min y enfriada a temperatura ambiente, para luego ser homogenizada (Ultraturrax IKA T25), a 12.000rpm, por $5 \mathrm{~min}$, adicionando el AV y la mezcla de hidrocoloides en las cantidades definidas, para cada tratamiento del diseño experimental. Finalmente, se agregaron los conservantes $(0,125 \%)$ y las bebidas fueron envasadas para su caracterización.

Caracterización fisicoquímica. Se realizó por triplicado para cada tratamiento, según los métodos de la AOAC (2016), determinando el porcentaje de sólidos solubles totales (SST), expresados en ${ }^{\circ}$ Brix; $\mathrm{pH}$, con el método potenciométrico; acidez expresada en porcentaje de ácido cítrico, por titulación con $\mathrm{NaOH} 0,1 \mathrm{~N}$ y, densidad, por el método gravimétrico.

Viscosidad aparente. Fue determinada con un viscosímetro (Brookfield DV-III Ultra, USA), utilizando una geometría de cilindros coaxiales, a $20^{\circ} \mathrm{C}$, y una tasa de deformación constante de $30 \mathrm{~s}^{-1}$ (Aghajanzadeh et al. 2017). La tasa de deformación fue seleccionada para simular condiciones de baja deformación del producto, como sucede durante el almacenamiento, por la sedimentación de partículas suspendidas.

Distribución del tamaño de partícula (PSD). Los parámetros $(\mathrm{D}[4,3])$ y $(\mathrm{D}[3,2])$ fueron determinados por el método de dispersión de luz (Malvern Mastersizer 3000 con Hydro EV), por triplicado, para cada tratamiento 


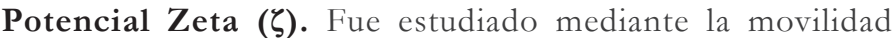
electroforética (Zetasizer nano ZS90, Malvern Instruments Ltd., UK), siguiendo el método descrito por Mirhosseini et al. (2008), con algunas modificaciones. Las muestras fueron diluidas en agua destilada ( $\mathrm{pH}=7)$, en una relación 1:50 y equilibradas durante 25s. Para cada tratamiento, se reportó el promedio de tres mediciones.

Estudio de separación de fases. La estabilidad conferida por los hidrocoloides a las bebidas formuladas fue evaluada a través de la separación de fases durante el almacenamiento, adaptando los métodos descritos por Silva et al. (2012). En cilindros graduados de $100 \mathrm{~mL}$ fueron colocadas las muestras cada tratamiento por triplicado y almacenadas a $10^{\circ} \mathrm{C}$, por 7 días, durante los cuales, se midió el cambio en la altura de la fase sedimentada. El índice de sedimentación fue calculado aplicando la ecuación 1 , donde, $\mathrm{h}(\mathrm{t})$ es la altura (mm) del sedimento en un tiempo $t(h)$ y ho la altura inicial (mm).

$$
I S=\frac{h(t)}{h_{0}} \times 100
$$

ecuación 1

La velocidad de sedimentación fue deducida con un modelo cinético de segundo orden (Ecuación 2), donde, IS (\%) es el índice de sedimentación, ISeq (\%) el índice de sedimentación en el equilibrio, $\mathrm{V}\left(\mathrm{h}^{-1}\right)$ es la velocidad de sedimentación y $\mathrm{t}$ (horas) es el tiempo.

$$
I S=I S e q\left(1-e^{-v t}\right)
$$

ecuación 2

Color. Los parámetros de color fueron medidos seguidamente a la elaboración de las bebidas, utilizando un espectofotómetro de esfera (X-Rite SP64, X-Rite Inc., USA), con iluminante D65 y ángulo de $10^{\circ}$. Los resultados fueron reportados en el sistema CIELab, para un promedio de tres mediciones por tratamiento, determinándose $\mathrm{L}^{*}$ (brillo), a* (componente rojo-verde), b* (componente amarilloazul) y la diferencia total de color $(\Delta \mathrm{E})$ respecto a la bebida control, mediante la ecuación 3.

$$
\Delta E=\sqrt{\left(\Delta L^{*}\right)^{2}+\left(\Delta a^{*}\right)^{2}+\left(\Delta b^{*}\right)^{2}}
$$

ecuación 3

Análisis sensorial. La evaluación sensorial de las bebidas fue conducida 15 horas después de su elaboración, a una temperatura aproximada de $10^{\circ} \mathrm{C}$, con el fin de establecer la percepción de la apariencia, el color, el sabor y el aroma. El panel fue realizado con 15 jueces semi-entrenados, aplicando una prueba descriptiva, paramétrica, con escala no estructurada (15cm de longitud), con anclajes en los extremos para cada parámetro evaluado. Para la apariencia, los anclajes fueron en relación a la separación de fases (Muy homogénea - Poco homogénea) y para los parámetros de color, sabor y aroma, los anclajes con respecto a las características del maíz dulce (Muy característico - Poco característico).

Análisis estadístico. Para estudiar las propiedades fisicoquímicas, sensoriales y de estabilidad, en función de la concentración de GX (-1: 0,025\%; 0: 0,05\%; 1: 0,075\%), CMC (-1: 0,025\%; 0: 0,05\%; 1: $0,075 \%)$ y AV (-1: $0,5 \% ; 0: 1 \% ; 1: 1,5 \%)$, fue aplicada la metodología de superficie de respuesta a un diseño central, compuesto de 18 tratamientos y 4 puntos centrales (Tabla 1). El tratamiento control fue la bebida formulada sin adición de hidrocoloides ni AV.

Las superficies de respuesta fueron calculadas mediante un modelo de segundo orden (Ecuación 4).

$$
Y=\beta_{0}+\sum_{i=1}^{3} \beta_{i} X_{i}+\sum_{i=1}^{3} \beta_{i i} X_{i}^{2}+\sum \sum_{i<j=1}^{3} \beta_{i j} X_{i} X_{j} \quad \text { ecuación } 4
$$

Donde, $Y$ es la respuesta calculada por el modelo, $\beta$, $\beta \mathrm{i}, \beta \mathrm{ii}$ y $\beta \mathrm{ij}$ son los coeficientes de regresión para el intercepto, términos de interacción lineales y cuadráticos, respectivamente y X es la variable independiente. La pertinencia de los modelos, se determinó con un ANAVA multifactorial $(\mathrm{p}=0,05)$, un análisis de regresión y una prueba de falta de ajuste.

\section{RESULTADOS Y DISCUSIÓN}

Caracterización fisicoquímica. Las propiedades fisicoquímicas de las bebidas, se muestran en la tabla 2. El contenido de SST, pH y acidez titulable no presentan variaciones significativas a diferentes concentraciones de hidrocoloides y AV ( $p>0,05)$. Estudios previos coinciden en que la adición de hidrocoloides a jugos, néctares y concentrados de frutas no produce cambios significativos en estas propiedades (Lins et al. 2014; Taiwo \& Gift, 2015). Elbandy et al. (2014), también manifiestan que la adición de AV en bebidas puede afectar levemente o no influye en la variación de los SST, debido a que el contenido de sólidos en el gel es insuficiente $(<5 \%)$, para producir modificaciones notables en el producto.

Viscosidad. La concentración de GX y CMC mostró tener un efecto significativo $(\mathrm{p}<0,05)$ sobre la viscosidad aparente de las bebidas (Tabla 2). Como se evidencia en la figura 1a, existe una relación entre la viscosidad y la concentración de hidrocoloides, lo cual, indica un cambio en el comportamiento reológico de la fase continua, como se ha demostrado anteriormente (Contreras-Lozano et al. 2018; Cho \& Yoo, 2015). Asimismo, se ha encontrado que la GX confiere altas viscosidades a bajas tasas de deformación, características que contribuyen a la estabilidad de partículas en suspensión (Norton et al. 2011). Los hidrocoloides producen este efecto, debido a que su estructura isotrópica rígida sufre interacciones moleculares con el agua durante la dispersión, provocando un aumento de la viscosidad aparente, característico de polímeros de alto peso molecular en suspensión (Xu et al. 2013).

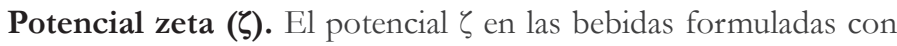
estabilizantes y el tratamiento control (Tabla 2), se encuentran en el rango de estabilidad ligera $(-21$ a $-30 \mathrm{mV})$, característico de poca aglomeración de partículas en suspensión (Schramm, 2005). Por su parte, el potencial $\zeta$ no presentó cambios significativos ( $p>0,05)$ en función de la concentración de hidrocoloides, en concordancia con lo reportado por Mirhosseini et al. (2008), en bebidas estabilizadas con GX y goma arábiga, manifestando que un rango de concentraciones, insuficientemente amplio, no produce una variación significativa de este parámetro. Es posible que las concentraciones de GX y CMC usadas en este estudio, en las 
Tabla 1. Diseño experimental.

\begin{tabular}{|c|c|c|c|c|c|}
\hline \multirow{2}{*}{ No. } & \multicolumn{3}{|c|}{ Niveles codificados } & \multicolumn{2}{c|}{ Concentración (\%) } \\
\cline { 2 - 6 } & AV & GX & CMC & AV & GX \\
\hline 1 & 0 & 0 & 1,68179 & 1 & 0,05 \\
\hline 2 & 0 & $-1,68$ & 0 & 1 & 0,008 \\
\hline 3 & $-1,68$ & 0 & 0 & 0,16 & 0,05 \\
\hline 4 & -1 & 1 & 1 & 0,5 & 0,075 \\
\hline 5 & 1 & -1 & 1 & 1,5 & 0,025 \\
\hline 6 & 0 & 0 & 0 & 1 & 0,05 \\
\hline 7 & 1 & 1 & -1 & 1,5 & 0,075 \\
\hline 8 & 0 & 0 & 0 & 1 & 0,05 \\
\hline 9 & 1 & -1 & -1 & 1,5 & 0,025 \\
\hline 10 & 0 & 0 & $-1,68$ & 1 & 0,05 \\
\hline 11 & 0 & 1,68 & 0 & 1 & 0,09 \\
\hline 12 & 1,68 & 0 & 0 & 1,84 & 0,05 \\
\hline 13 & 0 & 0 & 0 & 1 & 0,05 \\
\hline 14 & 0 & 0 & 0 & 1 & 0,05 \\
\hline 15 & -1 & -1 & 1 & 0,5 & 0,025 \\
\hline 16 & 1 & 1 & 1 & 1,5 & 0,075 \\
\hline 17 & -1 & -1 & -1 & 0,5 & 0,025 \\
\hline 18 & -1 & 1 & -1 & 0,5 & 0,075 \\
\hline
\end{tabular}

Tabla 2. Propiedades fisicoquímicas de las bebidas formuladas.

\begin{tabular}{|c|c|c|c|c|c|c|c|c|}
\hline No. & SST $\left({ }^{\circ}\right.$ Brix) & $\mathrm{pH}$ & Densidad $(\mathrm{g} / \mathrm{mL})$ & Acidez (\%) & Viscosidad (Pa.s) & Potencial $\zeta(\mathrm{mV})$ & $\mathrm{D}[3,2](\mu \mathrm{m})$ & $\mathrm{D}[4,3](\mu \mathrm{m})$ \\
\hline 1 & $10,33 \pm 0,29$ & $6,70 \pm 0,02$ & $1,0449 \pm 0,0003$ & $0,1293 \pm 0,0178$ & $25.14 \pm 0,44$ & $-25,25 \pm 0,07$ & $6,02 \pm 0,10$ & $9,65 \pm 0,89$ \\
\hline 2 & $9,467 \pm 0,21$ & $6,83 \pm 0,01$ & $1,0439 \pm 0,0009$ & $0,1340 \pm 0,0072$ & $7.49 \pm 0,29$ & $-27,35 \pm 0,21$ & $6,22 \pm 0,04$ & $11,30 \pm 0,44$ \\
\hline 3 & $10,33 \pm 0,15$ & $6,82 \pm 0,02$ & $1,0454 \pm 0,0010$ & $0,1329 \pm 0,0093$ & $23.32 \pm 0,76$ & $-25,65 \pm 0,35$ & $6,04 \pm 0,02$ & $9,22 \pm 0,05$ \\
\hline 4 & $10,40 \pm 0,66$ & $6,68 \pm 0,01$ & $1,0454 \pm 0,0002$ & $0,1321 \pm 0,0048$ & $33.59 \pm 0,72$ & $-23,55 \pm 0,21$ & $6,07 \pm 0,04$ & $10,50 \pm 0,49$ \\
\hline 5 & $10,33 \pm 0,12$ & $6,88 \pm 0,01$ & $1,0448 \pm 0,0009$ & $0,1320 \pm 0,0073$ & $16.31 \pm 0,17$ & $-25,70 \pm 0,28$ & $6,10 \pm 0,04$ & $10,80 \pm 0,30$ \\
\hline 6 & $9,70 \pm 0,40$ & $6,61 \pm 0,01$ & $1,0437 \pm 0,0004$ & $0,1116 \pm 0,0114$ & $22.94 \pm 0,72$ & $-23,75 \pm 0,07$ & $6,18 \pm 0,28$ & $12,90 \pm 0,59$ \\
\hline 7 & $9,93 \pm 0,06$ & $6,86 \pm 0,01$ & $1,0450 \pm 0,0006$ & $0,1323 \pm 0,0083$ & $29.37 \pm 0,29$ & $-26,13 \pm 0,32$ & $6,09 \pm 0,03$ & $8,99 \pm 0,10$ \\
\hline 8 & $9,93 \pm 0,06$ & $6,88 \pm 0,01$ & $1,0449 \pm 0,0009$ & $0,1129 \pm 0,0182$ & $22.27 \pm 0,17$ & $-27,00 \pm 0,28$ & $6,15 \pm 0,02$ & $9,48 \pm 0,15$ \\
\hline 9 & $9,80 \pm 0,26$ & $6,86 \pm 0,01$ & $1,0445 \pm 0,0008$ & $0,1034 \pm 0,0041$ & $9.64 \pm 0,54$ & $-21,60 \pm 0,10$ & $6,25 \pm 0,02$ & $11,30 \pm 0,27$ \\
\hline 10 & $10,13 \pm 0,06$ & $6,86 \pm 0,01$ & $1,0442 \pm 0,0006$ & $0,1066 \pm 0,0080$ & $15.83 \pm 0,29$ & $-24,75 \pm 0,21$ & $6,99 \pm 0,27$ & $14,90 \pm 0,84$ \\
\hline 11 & $10,83 \pm 0,21$ & $6,80 \pm 0,01$ & $1,0453 \pm 0,0007$ & $0,1325 \pm 0,0096$ & $42.22 \pm 0,20$ & $-24,20 \pm 0,28$ & $7,21 \pm 0,04$ & $18,30 \pm 0,48$ \\
\hline 12 & $10,10 \pm 0,10$ & $6,90 \pm 0,02$ & $1,0465 \pm 0,0009$ & $0,1327 \pm 0,0069$ & $24.28 \pm 0,93$ & $-24,20 \pm 0,42$ & $6,89 \pm 0,02$ & $14,40 \pm 0,71$ \\
\hline 13 & $9,06 \pm 0,23$ & $6,87 \pm 0,02$ & $1,0456 \pm 0,0007$ & $0,1226 \pm 0,0013$ & $25.24 \pm 0,44$ & $-25,80 \pm 0,71$ & $6,95 \pm 0,01$ & $15,00 \pm 0,49$ \\
\hline 14 & $9,86 \pm 0,25$ & $6,90 \pm 0,01$ & $1,0460 \pm 0,0011$ & $0,1250 \pm 0,0011$ & $24.57 \pm 0,60$ & $-25,65 \pm 0,07$ & $6,92 \pm 0,02$ & $15,20 \pm 0,46$ \\
\hline 15 & $9,73 \pm 0,06$ & $6,95 \pm 0,01$ & $1,0473 \pm 0,0008$ & $0,1324 \pm 0,0067$ & $19.29 \pm 0,50$ & $-23,85 \pm 0,49$ & $6,81 \pm 0,01$ & $13,80 \pm 0,09$ \\
\hline 16 & $10,03 \pm 0,51$ & $6,70 \pm 0,01$ & $1,0440 \pm 0,0004$ & $0,1242 \pm 0,0038$ & $33.78 \pm 0,60$ & $-23,30 \pm 0,01$ & $7,12 \pm 0,03$ & $20,00 \pm 0,46$ \\
\hline 17 & $10,26 \pm 0,25$ & $6,92 \pm 0,01$ & $1,0473 \pm 0,0010$ & $0,1242 \pm 0,0042$ & $11.71 \pm 0,44$ & $-25,35 \pm 0,07$ & $6,87 \pm 0,01$ & $14,30 \pm 0,71$ \\
\hline 18 & $9,86 \pm 0,25$ & $6,77 \pm 0,01$ & $1,0479 \pm 0,0005$ & $0,1219 \pm 0,0015$ & $32.15 \pm 0,16$ & $-22,10 \pm 0,28$ & $6,90 \pm 0,01$ & $14,40 \pm 0,06$ \\
\hline $\mathrm{C}$ & $10,50 \pm 0,50$ & $6,98 \pm 0,01$ & $1,0432 \pm 0,0007$ & $0,1244 \pm 0,0044$ & $2.9 \pm 0,27$ & $-24,10 \pm 0,24$ & $6,02 \pm 0,10$ & $9,65 \pm 0,70$ \\
\hline
\end{tabular}



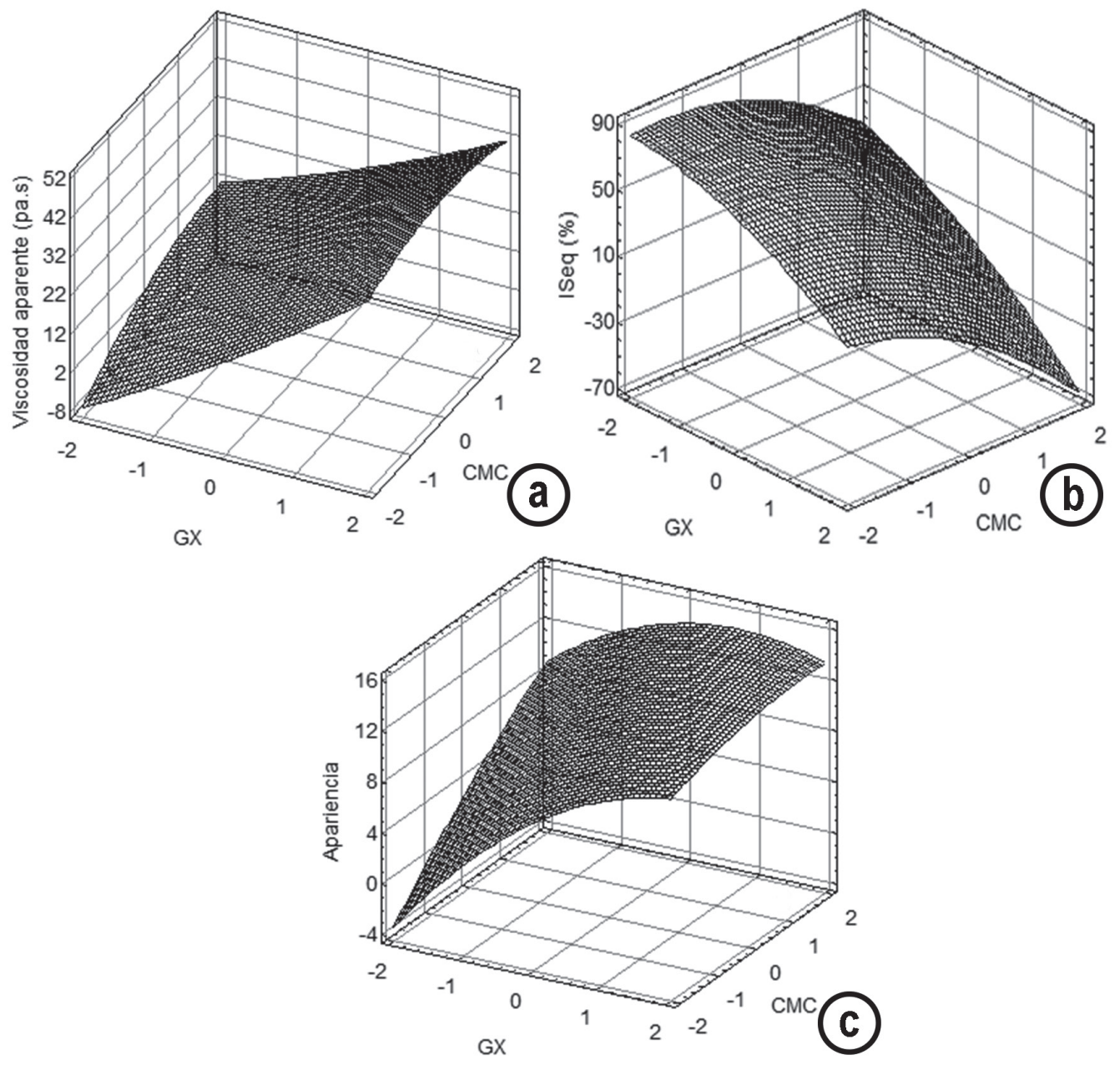

Figura 1. Superficies de respuesta con variación significativa $(\mathrm{p}<0,05)$. a) Viscosidad aparente; b) Índice de sedimentación en el equilibrio; c) Apariencia. La correspondencia de los niveles codificados para los factores es $(-1): 0,025 \% ;(0): 0,05 \% ;(1): 0,075 \%$.

bebidas de maíz, no fueron suficientemente altas para conferir buena estabilidad, expresada en valores de potencial $>40 \mathrm{mV}$ (Schramm, 2005), a diferencia de estudios similares, donde esa estabilidad fue alcanzada con concentraciones de GX $\geq 0,3 \%$ (Abbasi \& Mohammadi, 2013; Genovese \& Lozano, 2001). Una evidencia de esto es que en los tratamientos 1 y 8 se encontraron los resultados más favorables de potencial $\zeta$ y corresponden a los porcentajes mayores de hidrocoloides (GX y $\mathrm{CMC} \geq 0,05 \%$ ) y $1 \% \mathrm{AV}$.

Es posible que los valores encontrados de potencial $\zeta$ las bebidas de maíz se deban a la presencia de una fase oleosa, formada por las grasas del maíz y el ingrediente lácteo adicionado en la formulación, confiriéndoles el carácter de una emulsión $\mathrm{O} / \mathrm{W}$, que no se logró estabilizar con las concentraciones aplicadas de hidrocoloides. Esto se explica porque un valor absoluto de potencial $\zeta$ menor de $25 \mathrm{mV}$, como se observó en las bebidas, es un indicativo de floculación en emulsiones con tendencia hacia la coalescencia o cremado (Schramm, 2005). Adicionalmente, se ha reportado incompatibilidad de la GX con las proteínas de la leche y bajas concentraciones pueden tener un efecto desestabilizador en emulsiones (Norton et al. 2011; Dickinson, 2009).
Distribución del tamaño de partícula (PSD). El tamaño de partículas mostró una distribución mono modal entre $\sim 1$ y $600 \mu \mathrm{m}$, similar para todas las bebidas (Figura 2). Esto se debe a que, el proceso de homogenización, fue estandarizado para todas las formulaciones después de la adición de hidrocoloides. Este hallazgo, se confirma con el resultado del ANAVA, donde se reporta que no hay efecto significativo $(\mathrm{p}>0,05)$ de los factores sobre los diámetros $\mathrm{D}[3,2]$ y $\mathrm{D}[4,3]$.

El diámetro medio basado en el área $\mathrm{D}[3,2]$ fue inferior al rango del diámetro medio basado en el volumen D[4,3] (Tabla 2). Dado que, el primero, está influenciado por pequeñas partículas que pueden ser las partículas sedimentables de almidón en la suspensión, mientras que, el segundo, está asociado a partículas de mayor tamaño en sistemas polidispersos, como pequeños glóbulos de aceite formados durante la homogeneización de las emulsiones (Drapala et al. 2018).

Se evidencia en la tabla 2, que la formulación con GX, CMC y AV aumentó el tamaño de partícula de algunas bebidas en relación al tratamiento control, posiblemente, porque los polisacáridos adicionados poseen fracciones insolubles que confieren grandes 


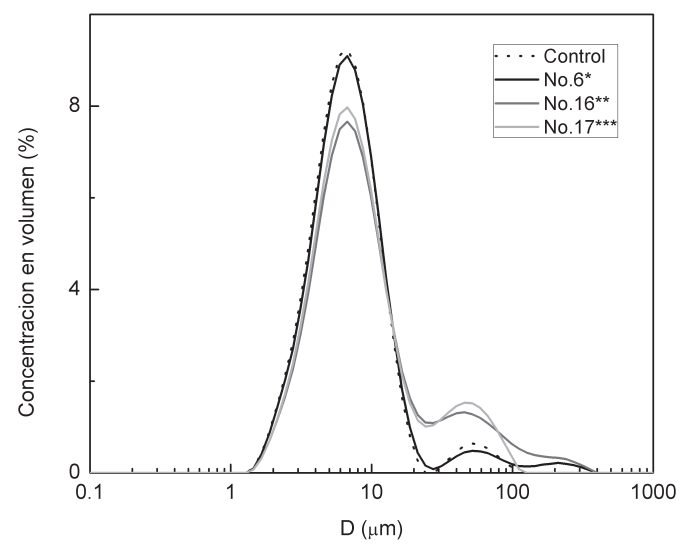

Figura 2. Distribución del tamaño de partícula en cuatro formulaciones de bebidas de maíz dulce. *Punto central (1\% AV - 0,05\% GX - 0,05\% CMC), **Punto axial superior (1,5\% AV - 0,075\% GX - 0,075\% CMC), ***Punto axial inferior (0,5\% AV- 0,025\% GX - 0,025\% CMC).

tamaños de partícula, incrementándose el diámetro medio (Ruihuan et al. 2017). Esto también puede ser una consecuencia de la floculación causada por la incompatibilidad entre la GX y la caseína láctea (Keshtkaran et al. 2013).

Se ha manifestado que las dispersiones con distribución de partículas con tendencia hacia tamaños pequeños $(<1 \mu \mathrm{m})$ tendrán mayor

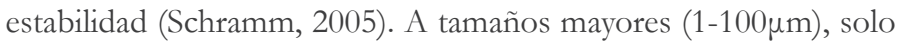
se lograría la estabilidad de una bebida adicionando concentraciones adecuadas de estabilizantes. De esta manera, los diámetros de partícula alcanzados, después de la homogenización de las bebidas (6-20 $\mu \mathrm{m})$, podrían ser adecuados para mantener la estabilidad a la sedimentación, si se adicionan mayores concentraciones de GX y se reduce el tamaño de las gotas que componen la emulsión, aplicando un tratamiento de homogenización adicional.

Estudio de separación de fases. Se observó, en la mayoría de las bebidas formuladas, la formación de tres fases; una inferior, con mayor concentración de partículas (sedimento); una clarificada, con menor concentración de sólidos (sobrenadante) y una oleosa, en la parte superior del cilindro graduado, lo que demuestra que las concentraciones de hidrocoloides adicionadas no resultaron efectivas para estabilizar la emulsión, en concordancia los valores de potencial $\zeta$ (Tabla 2).

Los datos de sedimentación fueron ajustados a un modelo cinético de primer orden (Figura 3), que resultó ser adecuado $\left(\mathrm{R}^{2}>0,90\right)$ para todos los tratamientos que presentaron sedimentación. La concentración de GX y CMC produjo un efecto significativo $(\mathrm{p}<0,05)$ en el parámetro ISeq, el cual, disminuye a mayor concentración de hidrocoloides (Figura 1b). Por el contrario, la concentración de AV no tuvo ningún efecto. Se ha demostrado que la adición de GX y CMC mejoran la estabilidad física reduciendo la sedimentación de partículas en bebidas por el incremento de la viscosidad del medio (Taiwo \& Gift, 2015; Akkarachaneeyakorn \& Tinrat, 2015).

La velocidad de sedimentación de las bebidas formuladas con GX, CMC y AV $\left(0-0,32 \mathrm{~h}^{-1}\right)$ fue notablemente inferior que en la bebida con tratamiento control $\left(3,13 \mathrm{~h}^{-1}\right)$. De esta forma, se evidencia la efectividad de la adición de hidrocoloides para

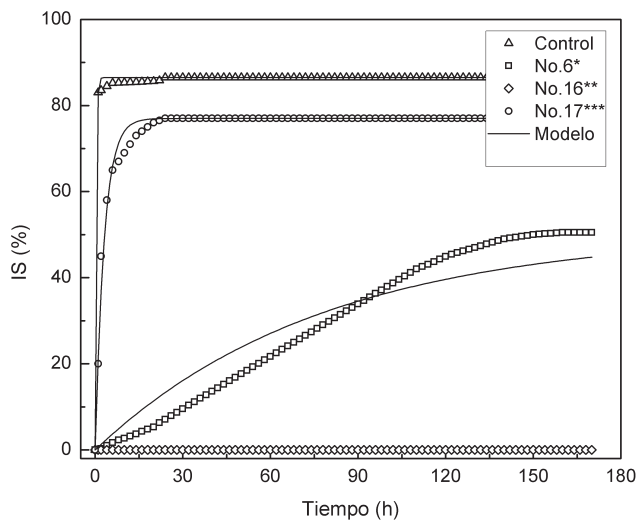

Figura 3. Ajuste del índice de sedimentación al modelo cinético. *Punto central (1\% AV - 0,05\% GX - 0,05\% CMC), **Punto axial superior (1,5\% AV - 0,075\% GX - 0,075\% CMC), ***Punto axial inferior (0,5\% AV- 0,025\% GX - 0,025\% CMC). 
retrasar la separación de fases por sedimentación en las bebidas durante el almacenamiento en refrigeración $\left(10^{\circ} \mathrm{C}\right)$. Los tratamientos correspondientes a mayor concentración de hidrocoloides (No. 16), principalmente de GX (No. 11), no presentaron sedimentación. Además, los tratamientos No. 1 y No. 4 mostraron los más bajos índices de sedimentación. En consecuencia, se concluye que es posible disminuir el índice de sedimentación hasta 7 días en refrigeración aplicando concentraciones mínimas de 0,05\% de GX y $0,075 \%$ de CMC.

Color. En la tabla 3, se muestran los parámetros de color CIELab y la diferencia total de color $(\Delta \mathrm{E})$ en las bebidas formuladas, los cuales, no presentaron variación estadística significativa $(\mathrm{p}>0,05)$ con la concentración de hidrocoloides y AV. Los valores del parámetro $a^{*}$ y b* expresan que el color de las bebidas tiende hacia el amarillo y verde. Estos no presentaron diferencias considerables respecto al control, a diferencia de la luminosidad L*, que aumentó luego de la adición de hidrocoloides y AV. Posiblemente, el color blanco o amarillento, característico de los hidrocoloides, otorga una apariencia más clara y brillante. Esta alteración de la luminosidad también podría estar asociada a la variación del tamaño de partícula por formación de agregados de mayor tamaño, lo que puede afectar el patrón de difracción (Keshtkaran et al. 2013). En correspondencia con los parámetros primarios, la diferencia total de color $(\Delta \mathrm{E})$ en las bebidas tratadas con hidrocoloides respecto al tratamiento control fue baja y no fue significativa $(p>0,05)$, con relación a la concentración.

Análisis sensorial. La tabla 3 contiene los resultados de la evaluación sensorial de las bebidas formuladas. La concentración de GX y CMC influyó significativamente en la apariencia de bebidas y el ajuste al modelo cuadrático resultó significativo para esta variable
$\left(\mathrm{R}^{2}=0,89\right)$. La calificación otorgada a la apariencia es más alta en las bebidas con mayor concentración de hidrocoloides, como se observa en la superficie de respuesta (Figura 1c). Así mismo, la calificación de la apariencia de las bebidas con estabilizantes es mayor que en la muestra control. Lo resultados, se justifican por la relación directa que tiene la apariencia con la homogeneidad percibida en las bebidas, es decir, con la separación de fases, que es menor luego de la adición de hidrocoloides. Taiwo \& Gift (2015), también encontraron diferencias significativas en la percepción del nivel de sedimentación en jugos, luego de la adición de GX y CMC.

Las mayores puntuaciones de la apariencia fueron asignadas a los tratamientos No. 11 y No. 16, que fueron los únicos que no presentaron sedimentación durante el estudio de separación de fases.

Por su parte, la valoración sensorial del color, aroma y sabor, no varió significativamente con la concentración de hidrocoloides y AV. Además, las calificaciones obtenidas para estos parámetros fueron mayores en la bebida control (Tabla 3), es decir, que el color, sabor y aroma fue más característico del maíz dulce en la bebida sin estabilizantes. El efecto en la percepción de color puede estar relacionado con el incremento de la opacidad en presencia de hidrocoloides, en algunos casos, debido a partículas insolubles presentes o la incorporación de aire en la matriz alimentaria (Laaman, 2011). Por otro lado, se ha reportado que la intensidad del sabor puede disminuir, debido al uso de hidrocoloides en alimentos, pero que la concentración de las mismas no provoca diferencias notables en esta propiedad sensorial (Abedi et al. 2012). En cuanto al aroma, Keshtkaran et al. (2013) tampoco reportan variaciones con la concentración de hidrocoloides, mientras que Abedi et al. (2012) sostienen que el incremento de la viscosidad puede afectar la percepción del aroma en jugos lácteos formulados con estos aditivos,

Tabla 3. Parámetros de color y sensoriales de bebidas evaluadas.

\begin{tabular}{|c|c|c|c|c|c|c|c|c|}
\hline \multirow{2}{*}{ No. } & \multicolumn{4}{|c|}{ Parámetros de Color } & \multicolumn{4}{|c|}{ Evaluación de parámetros sensoriales } \\
\hline & $\mathrm{L}^{*}$ & $a^{*}$ & $b^{*}$ & $\Delta \mathrm{E}$ & Apariencia & Color & Aroma & Sabor \\
\hline 1 & $72,003 \pm 0,032$ & $-1,747 \pm 0,020$ & $19,623 \pm 0,015$ & $1,059 \pm 0,018$ & $13,2 \pm 2,6$ & $12,8 \pm 2,7$ & $8,7 \pm 2,7$ & $9,0 \pm 2,8$ \\
\hline 2 & $70,963 \pm 0,087$ & $-1,833 \pm 0,006$ & $19,450 \pm 0,042$ & $2,142 \pm 0,088$ & $5,1 \pm 2,5$ & $11,0 \pm 2,5$ & $6,6 \pm 0,1$ & $6,6 \pm 2,2$ \\
\hline 3 & $71,717 \pm 0,075$ & $-1,717 \pm 0,012$ & $19,553 \pm 0,020$ & $1,075 \pm 0,068$ & $11,4 \pm 2,4$ & $13,0 \pm 2,1$ & $6,7 \pm 0,8$ & $8,8 \pm 3,1$ \\
\hline 4 & $71,167 \pm 0,086$ & $-1,670 \pm 0,023$ & $20,003 \pm 0,139$ & $1,185 \pm 0,065$ & $13,7 \pm 0,9$ & $13,2 \pm 1,7$ & $8,7 \pm 1,6$ & $9,8 \pm 1,6$ \\
\hline 5 & $70,483 \pm 0,147$ & $-1,713 \pm 0,015$ & $19,793 \pm 0,070$ & $1,552 \pm 0,155$ & $10,3 \pm 4,3$ & $12,8 \pm 2,7$ & $8,2 \pm 2,5$ & $8,4 \pm 2,3$ \\
\hline 6 & $70,953 \pm 0,145$ & $-1,707 \pm 0,031$ & $19,617 \pm 0,081$ & $1,439 \pm 0,151$ & $12,9 \pm 2,7$ & $13,2 \pm 1,5$ & $7,7 \pm 1,5$ & $8,0 \pm 1,6$ \\
\hline 7 & $70,363 \pm 0,578$ & $-1,733 \pm 0,093$ & $19,633 \pm 0,436$ & $2,007 \pm 0,717$ & $13,1 \pm 2,7$ & $13,1 \pm 2,1$ & $8,8 \pm 2,8$ & $10,0 \pm 2,6$ \\
\hline 8 & $70,437 \pm 0,325$ & $-1,703 \pm 0,062$ & $19,713 \pm 0,146$ & $1,571 \pm 0,342$ & $9,8 \pm 2,3$ & $12,9 \pm 0,5$ & $6,1 \pm 2,2$ & $6,6 \pm 1,1$ \\
\hline 9 & $70,610 \pm 0,172$ & $-1,627 \pm 0,045$ & $19,913 \pm 0,133$ & $1,212 \pm 0,202$ & $3,8 \pm 1,7$ & $12,8 \pm 2,3$ & $8,9 \pm 1,9$ & $9,8 \pm 2,0$ \\
\hline 10 & $70,103 \pm 0,689$ & $-1,743 \pm 0,035$ & $19,853 \pm 0,614$ & $2,056 \pm 0,738$ & $9,3 \pm 1,5$ & $11,8 \pm 2,0$ & $12,6 \pm 1,9$ & $10,5 \pm 1,8$ \\
\hline 11 & $71,543 \pm 0,100$ & $-1,700 \pm 0,056$ & $20,553 \pm 0,166$ & $1,752 \pm 0,145$ & $14,1 \pm 2,2$ & $13,6 \pm 2,9$ & $8,3 \pm 1,7$ & $8,5 \pm 1,8$ \\
\hline 12 & $72,897 \pm 1,818$ & $-1,387 \pm 0,085$ & $21,550 \pm 0,833$ & $1,185 \pm 1,087$ & $13,2 \pm 1,9$ & $9,8 \pm 2,3$ & $6,5 \pm 2,1$ & $7,0 \pm 1,1$ \\
\hline 13 & $73,110 \pm 0,175$ & $-1,530 \pm 0,067$ & $22,043 \pm 0,165$ & $1,077 \pm 0,168$ & $12,5 \pm 2,3$ & $13,0 \pm 2,0$ & $7,0 \pm 1,0$ & $7,3 \pm 2,6$ \\
\hline 14 & $73,383 \pm 0,166$ & $-1,507 \pm 0,020$ & $22,230 \pm 0,148$ & $0,777 \pm 0,090$ & $10,0 \pm 2,4$ & $13,5 \pm 1,4$ & $7,5 \pm 1,6$ & $7,8 \pm 2,2$ \\
\hline 15 & $72,373 \pm 0,311$ & $-1,633 \pm 0,015$ & $21,327 \pm 0,110$ & $1,000 \pm 0,282$ & $11,6 \pm 1,4$ & $10,4 \pm 2,3$ & $11,5 \pm 1,4$ & $11,6 \pm 1,6$ \\
\hline 16 & $71,450 \pm 0,147$ & $-1,710 \pm 0,057$ & $20,140 \pm 0,151$ & $1,841 \pm 0,163$ & $14,6 \pm 0,9$ & $10,5 \pm 1,1$ & $7,8 \pm 1,8$ & $6,0 \pm 2,2$ \\
\hline 17 & $73,187 \pm 0,609$ & $-1,477 \pm 0,023$ & $21,830 \pm 0,366$ & $1,035 \pm 0,685$ & $6,5 \pm 2,2$ & $14,0 \pm 0,8$ & $13,5 \pm 1,2$ & $9,5 \pm 1,0$ \\
\hline 18 & $73,610 \pm 1,161$ & $-1,500 \pm 0,055$ & $22,335 \pm 0,437$ & $1,291 \pm 1,120$ & $8,7 \pm 1,3$ & $9,5 \pm 1,8$ & $11,1 \pm 2,5$ & $12,6 \pm 2,9$ \\
\hline $\mathrm{C}$ & $47,107 \pm 0,023$ & $-1,620 \pm 0,006$ & $20,000 \pm 0,061$ & - & $4,8 \pm 0,5$ & $14,1 \pm 2,1$ & $13,9 \pm 2,6$ & $13,3 \pm 2,8$ \\
\hline
\end{tabular}


lo que, posiblemente, ocurrió en las bebidas de maíz luego de la estabilización, debido al incremento de la viscosidad.

Del presente estudio, se puede concluir que las propiedades fisicoquímicas en las bebidas formuladas a partir de maíz dulce no son afectadas con las concentraciones aplicadas de AV e hidrocoloides. La inestabilidad física de las bebidas estudiadas, se evidencia en los valores de potencial z y está asociada con los fenómenos de sedimentación de partículas suspendidas e inestabilidad de la emulsión formada por causa de la fase grasa observada. La inestabilidad podría ser consecuencia de la adición de GX en altas concentraciones, promoviendo la floculación de la fase oleosa. La adición de hidrocoloides favorece la estabilidad a la sedimentación a concentraciones mayores de 0,05\% de GX y CMC e, independientemente, de la concentración de AV. El tamaño de partículas en las bebidas formuladas no resultó adecuado para conferir la estabilidad. La adición de hidrocoloides y AV aumenta la luminosidad de las bebidas y favorece la percepción de la apariencia en las bebidas con menor sedimentación durante el almacenamiento. El color, el sabor y el aroma fueron más valorados en la bebida sin adición de hidrocoloides.

Agradecimientos: A la Dirección de Investigación y Extensión (DIME) -Universidad Nacional de Colombia - sede Medellín, por la financiación de esta investigación. Conflicto de intereses: El manuscrito fue preparado y revisado con la participación de todos los autores, quienes declaramos que no existe ningún conflicto de intereses que ponga en riesgo la validez de los resultados presentados.

\section{REFERENCIAS}

1. ABBASI, S.; MOHAMMADI, S. 2013. Stabilization of milkorange juice mixture using Persian gum: Efficiency and mechanism. Food Bioscience. (Reino Unido). 2(1):53-60. https://doi.org/10.1016/j.fbio.2013.04.002

2. ABEDI, F.; SANI, A.M.; KARAZHIYAN, H. 2012. Effect of some hydrocolloids blend on viscosity and sensory properties of raspberry juice milk. J. Food Science and Technology. (India). 51(9):2246-2250. https://doi. org/10.1007/s13197-012-0705-0

3. AGHAJANZADEH, S.; MOHAMMAD Z.; KASHANINEJAD, M. 2017. Influence of thermal treatment, homogenization and xanthan gum on physicochemical properties of watermelon juice: A response Surface approach. LWT Food Science and Technology. (Inglaterra). 85(1):66-74. https://doi.org/10.1016/j.lwt.2017.07.007

4. AKKARACHANEEYAKORN, S.; TINRAT, S. 2015. Effects of types and amounts of stabilizers on physical and sensory characteristics of cloudy ready-to-drink mulberry fruit juice. Food Science and Nutrition. (USA). 3(3):213-220. https://doi.org/10.1002/fsn3.206
5. ASSOCIATION OF OFFICIAL ANALYTICAL CHEMISTS, AOAC. 2016. Official Methods of Analysis. 20 ed. Ed. AOAC International.

6. CHO, H.M.; YOO, B. 2015. Rheological characteristics of cold thickened beverages containing xanthan gume based food thickeners used for dysphagia diets. J. Academy of Nutrition and Dietetics. (USA). 115(1):106-111. https:// doi.org/10.1016/j.jand.2014.08.028

7. CONTRERAS-LOZANO, K.; CIRO-VELASQUEZ, H.; MARQUEZ-CARDOZO. 2018. Effect of the addition of hydrocolloids and aloe vera gel (Aloe barbadensis Miller) on the rheological properties of a beverage of sweet corn (Zea mays var. saccharata). DYNA. (Colombia). 85(204):302-310. https://doi.org/10.15446/dyna.v85n204.63205

8. DICKINSON, E. 2009. Hydrocolloids as emulsifiers and emulsion stabilizers. Food Hydrocolloids. (Holanda). 23(6):1473-1482. https://doi.org/10.1016/j. foodhyd.2008.08.005

9. DRAPALA, K.P.; MULVIHILL, D.M.; O’MAHONY, J.A. 2018. A review of the analytical approaches used for studying the structure, interactions and stability of emulsions in nutritional beverage systems. Food Structure. (Holanda). 16(1):27-42. https://doi.org/10.1016/j.foostr.2018.01.004

10. ELBANDY, M.A.; ABED, S.M.; GAD, S.S.; ABDEL, F.M. 2014. Aloe vera gel as a functional ingredient and natural preservative in mango nectar. World J. Dairy \& Food Sciences. (Egipto). 9(2):191-203. https:/ / doi.org/10.5829/ idosi.wjdfs.2014.9.2.1139

11. GENOVESE, D.B.; LOZANO, J.E. 2001. The effect of hydrocolloids on the stability and viscosity of cloudy apple juices. Food Hydrocolloids. (Holanda). 15(1):1-7. https:// doi.org/10.1016/S0268-005X(00)00053-9

12. KESHTKARAN, M.; MOHAMMADIFAR, M.A.; ASADI, G.H.; NEJAD, R.A. 2013. Effect of gum tragacanth on rheological and physical properties of a flavored milk drink made with date syrup. J. Dairy Science. (USA). 96(8):4794803. https://doi.org/10.3168/jds.2012-5942

13. LAAMAN, T.R. 2011. Hydrocolloids: Fifteen Practical Tips. En: Laaman, T.R. (Ed). Hydrocolloids in Food Processing. Ed. Blackwell Publishing, Ltd. (Iowa). p.1-17.

14. LINS, A.C.; CAVALCANTI, D.T.; AZOUBEL, P.M.; MÉLO, E.; MACIEL, M.I. 2014. Effect of hydrocolloids on the physicochemical characteristics of yellow mombin structured fruit. Food Science and Technology (Brasil). 34(3):456-463. https://doi.org/10.1590/1678-457x.6348 
15. MIRHOSSEINI, H.; TAN, C.P.; HAMID, N.S.A.; YUSOF, S. 2008. Effect of Arabic gum, xanthan gum and orange oil contents on $\zeta$-potential, conductivity, stability, size index and $\mathrm{pH}$ of orange beverage emulsion. Colloids and Surfaces A: Physicochemical and Engineering Aspects. (Holanda). 315(1):47-56. https://doi.org/10.1016/j. colsurfa.2007.07.007

16. NORTON, I.; SPYROPOULOS, F.; COX, P. 2011. Practical Food Rheology. Ed. Blackwell Publishing Ltd. (Oxford). 278p.

17. NWAOKORO, O.G.; AKANBI, C.T. 2015. Effect of the addition of hydrocolloids to tomatocarrot juice blend. J. Nutritional Health \& Food Science. 3(1):1-10. http:// dx.doi.org/10.15226/jnhfs.2015.00134

18. RUIHUAN, L.; QING, K.; HAIJIN, M.; XIAODAN F. 2017. Effect of guar gum on stability and physical properties of orange juice. Internal J. Biological Macromolecules. (Holanda). 98(1):565-574. https://doi.org/10.1016/j. ijbiomac.2017.02.031

19. SÁNCHEZ-MACHADO, D.; LÓPEZ-CERVANTES, J.; SENDÓN, R.; SANCHES-SILVA, A. 2017. Aloe vera: ancient knowledge with new frontiers. Trends in Food Science \& Technology (Holanda). 61(1):94-102. https:// doi.org/10.1016/j.tifs.2016.12.005
20. SCHRAMM, L.L. 2005. Emulsions, Foams, and Suspensions: Fundamentals and Applications. Ed. Wiley-VCH Verlag GmbH y Co. KGaA (Weinheim). 465p.

21. SILVA, V.M.; SATO, A.C.; BARBOSA, A.C.; DACANAL, G.; CIRO-VELÁSQUEZ, H.J.; CUNHA, R.L. 2012. The effect of homogenisation on the stability of pineapple pulp. Internal J. Food Science and Technology. (USA). 45(10):2127-2133. https://doi.org/10.1111/j.13652621.2010.02386.x

22. SZYMANEK, M.; TANAŚ, W.; HAMMED, K. 2015. Kernel carbohydrates concentration in sugary-1, sugary enhanced and shrunken sweet corn kernels. Agriculture and Agricultural Science Procedia. (Holanda). 7(1):260-264. https://doi.org/10.1016/j.aaspro.2015.12.044

23. TAIWO, A.C.; GIFT, N.O. 2015. Effect of the addition of hydrocolloids to tomato-carrot juice blend. J. Nutritional Health \& Food Science (USA). 3(1):1-10. https://doi. org/10.4172/2155-9600.1000212

24. XU, L.; XU, G.; LIU, T.; CHEN, Y.; GONG, H. 2013. The comparison of rheological properties of aqueous welan gum and xanthan gum solutions. Carbohydrate Polymers. (Reino Unido). 92(1):516-522. https://doi.org/10.1016/j. carbpol.2012.09.082 